\title{
The roles of CAM5.2 and caldesmon IHC stains in challenge of diagnosing adenoma, intramucosal carcinoma, and invasive adenocarcinoma on colon biopsies
}

\author{
Yue Jia, Ping Ji, Luan Nguyen, Felicita Baratelli and Samuel French* \\ Department of Pathology, Harbor-UCLA Medical Center, Torrance, CA, USA
}

\begin{abstract}
Making a distinction between adenoma, intramucosal carcinoma, and invasive carcinoma on small biopsies of colon polypoid lesions can occasionally pose a challenge to pathologists. In our study, we aimed to assess the staining patterns and utilities of the two immunohistochemical stains CAM5.2 and caldesmon in helping with this endeavor. Routinely processed colon polyps biopsies' specimens from 57 patients with CAM5.2 and caldesmon ordered were reviewed by three pathologists. We found CAM5.2 and caldesmon to be very useful in classifying these colon polypoid lesions. CAM5.2 highlighted single cells and cribriform architecture. Caldesmon stained the lamina propria, the smooth muscle, and the desmoplastic stroma surrounding invasive tumor in colonic submucosa with different morphologic characteristics. First, in the lamina propria, caldesmon was mostly negative with faint brown lines surrounding glands and small vessels. Second, in the smooth muscle of muscularis mucosa, caldesmon stained the cytoplasm lightly with solid and diffuse pattern. Third, in the desmoplastic area of the submucosa, caldesmon stained the cytoplasm strongly with a clear space or groove seen at the middle of the cytoplasm (tram-tracking appearance). We described our findings in this study with hope that more pathologists will utilize these two stains in their common practice to reliably diagnose invasion by colon carcinoma.
\end{abstract}

\section{Introduction}

Colon biopsies are very common pathological specimens in most pathology practices. The purposes of most colon biopsies are to rule out malignancies in polypoid lesions seen in the gastrointestinal tract during endoscopy. Per WHO Classification of Tumors of the Digestive System $4^{\text {th }}$ edition, lesions that are confined to the lamina propria without invasion through the muscularis mucosa into the submucosa have virtually no risk of metastasis. Thus, dysplastic lesions restricted to the mucosa (premalignant) are termed either adenoma (low grade intraepithelial neoplasia or LGIN) or intramucosal carcinoma (high grade intraepithelial neoplasia or HGIN). Dysplastic glandular lesions that invade into the submucosa (malignant) are termed invasive adenocarcinoma (or IC). Making the distinctions between these three entities (LGIN, HGIN and IC) is crucial because patients with HGIN or IC may require a colectomy rather than polypectomy. This important task can occasionally pose a challenge to pathologists, especially in small and fragmented biopsies.

Traditionally, HGIN is identified through finding architectural complexity and/or cytologic atypia that are more malignant-appearing than seen in LGIN [1]. High grade architecture changes show classic cribriform architecture with back-to-back crowded glands. Invasive carcinoma, on the other hand, is confirmed through assessing for desmoplasia or desmoplastic reaction in the stroma surrounding the dysplastic glands. Stromal fibroblasts surrounding tumors in the submucosa are activated and transformed to become myfibroblasts [2].

Immunohistochemical (IHC) stains have been very useful in determining the origin and differentiation of GI tract tumors [3]. However, very few publications are found regarding the roles of IHC stains in assisting to distinguish benign versus malignant colon polyps [4]. At our institution, we created a panel of stains consisting of CAM5.2 and caldesmon to help with this endeavor.

CAM5.2 is a low molecular weight cytokeratin stain that is commonly used to highlight epithelial cells. CAM5.2 has been reported to be useful for detecting lymph node micrometastasis or isolated tumor cells in various carcinomas [5].

Caldesmon is a protein that regulates myosin activity in smooth muscle cells. The role of caldesmon has been studied in both benign and malignant lesions. Per recent literature, the stain can be used to distinguish smooth muscle cells from other cells such as from myofibroblastic cells in skin lesions or stromal cells in endometrial stromal tumors [6,7]. Caldesmon has also been studied for its utility in differentiating between $\mathrm{pT} 3$ and $\mathrm{pT} 4$ colorectal cancer [8].

In our study, we assess the staining patterns and utilities of these two IHC stains in the aforementioned task. To the best of our knowledge, this is the first study where both CAM5.2 and caldesmon are evaluated simultaneously to assess their usefulness in distinguishing between high grade dysplasia and invasion of the colon polypoid malignancies.

${ }^{\star}$ Correspondence to: Samuel French, Department of Pathology, Harbor-UCLA Medical Center, Torrance, CA, USA, E-mail: sfrench@lundquist.org

Key words: colon biopsy, adenoma, intramucosal carcinoma, invasive adenocarcinoma, CAM5.2, caldesmon

Received: October 03, 2019; Accepted: October 21, 2019; Published: October 28, 2019 
Jia Y (2019) The roles of CAM5.2 and caldesmon IHC stains in challenge of diagnosing adenoma, intramucosal carcinoma, and invasive adenocarcinoma on colon biopsies

\section{Material and methods}

57 cases of colon polyps' biopsies with CAM5.2 and caldesmon ordered between January 2016 and July 2018 (period of 2.5 years) were found. Per our pathologists' consensus, the two IHC stains were ordered on cases where there was doubt on H\&E stained specimens whether the dysplastic cells showed high grade features or whether the dysplastic cells showed invasion into the lamina propria or the submucosa based on the induction of myofibroblasts.

Immunohistochemistry was performed on $4 \mu \mathrm{m}$-thick formalinfixed, paraffin-embedded tissue. The following antibodies were used: CAM5.2 (Roche, Pleasanton, CA), and caldesmon (clone E89; CellMarque, Pasadena, CA). Tissue sections were incubated with the primary antibody for a total of thirty minutes by Heat Induced Epitope Retrieval, subsequently washed with phosphate buffered saline, and then incubated with a secondary antibody that was conjugated with horseradish peroxidase. The whole staining process was done using Ventana Benchmark XT.

The 57 cases' slides were reviewed by three pathologists, one resident (LN) and two attendings (SF and PJ), to look for the findings of single cells, cribriform architecture, and desmoplastic patterns with assist of the IHC stains (Table 1). The cases were randomized and the reviewers did not know the cases' final pathology diagnosis.

\section{Results}

Out of 57 cases, 9 cases' the final diagnosis were LGIN, 16 cases were diagnosed as HGIN, and 32 cases were diagnosed as IC.

We found CAM5.2 and caldesmon to be very useful in classifying these colon polypoid lesions. CAM5.2 highlights single cells and cribriform architecture (Figure 1). Caldesmon stained the lamina propria, the smooth muscle, and desmoplastic stroma with different morphologic characteristics (Figure 2).

Caldesmon showed three different patterns. First, in the lamina propria, caldesmon was mostly negative with faint brown lines surrounding glands and small vessels. Second, in the smooth muscle of muscularis mucosa, caldesmon stained the cytoplasm lightly with solid and diffuse pattern. Third, in the desmoplastic area of the submucosa, caldesmon stained the cytoplasm strongly with the clear space or groove seen at the middle of the cytoplasm. The desmoplastic cells were closely packed and formed thickened bands of cells that ran parallel along the tumor cell borders.

Desmoplastic stroma consisted of proliferating myofibroblasts that indicate submucosal invasion. The proliferating myofibroblasts formed a slit-like unstained space in the middle of cytoplasm, whereas the smooth muscle cells stained throughout the cytoplasm as a solid sheet (Figures 2D, 2E, 2F).

No notable discrepancies were seen in interpretation between the three evaluators. All 9 cases of LGIN were negative for the three criteria evaluated (single cells, cribriform architecture and desmoplasia). 2 out of 16 cases of HGIN showed single cells and 16 out of 16 cases of HGIN showed cribriform architecture. 12 out of 32 cases of IC showed single cells, and 32 out of 32 cases of IC showed cribriform architecture. All 32

Table 1. Template that the pathologists used to evaluate the three criteria in 57 cases. The pathologists did not knowthe final diagnosis (blinded study). The presence of a positive finding was check-marked

\begin{tabular}{|c|c|c|c|}
\hline Case Number & Single cells & Cribriform pattern & Desmoplasia \\
\hline Example 1 & & $\sqrt{ }$ & \\
\hline
\end{tabular}

Table 2. Summary of result from pathologists reviewing 57 cases' slides

\begin{tabular}{|c|c|c|c|}
\hline & Single cells & Cribriform pattern & Desmoplasia \\
\hline LGIN $(9$ cases $)$ & $0 / 9(0 \%)$ & $0 / 9(0 \%)$ & $0 / 9(0 \%)$ \\
\hline HGIN $(16$ cases $)$ & $2 / 16(13 \%)$ & $16 / 16(100 \%)$ & $0 / 16(0 \%)$ \\
\hline IC $(32$ cases $)$ & $12 / 32(38 \%)$ & $32 / 32(100 \%)$ & $32 / 32(100 \%)$ \\
\hline Total $(57$ cases $)$ & & & \\
\hline
\end{tabular}
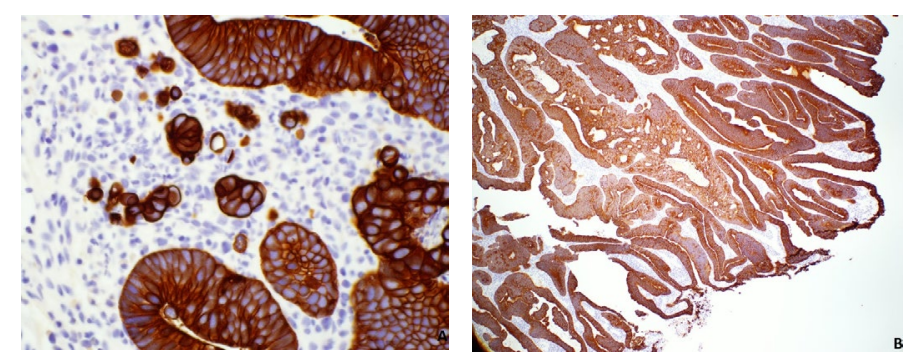

Figure 1. Immunostain of CAM5.2. A. CAM5.2 (40x) highlighted single cells in a case of HGIN. B. CAM5.2 (4x) highlighted cribriform architecture in a case of HGIN

cases of IC showed the strong tram-track pattern of desmoplasia which stained positive for caldesmon. All 9 cases of LGIN and 16 cases of HGIN were negative for this desmoplasia staining pattern. Frequency of positive findings are summarized in Table 2 .

Out of the 57 cases, we found the two HGIN cases showing single cells particularly interesting since finding single cells was usually a criteria in poorly differentiated carcinoma. Colectomy was done for one case and follow up colonoscopy was done for the other case. No residual tumor nor evidence of invasion was seen for subsequent specimens in both cases, consistent with intraepithelial neoplasm diagnosis.

Follow-ups for the remaining cases showed no significant discordance with the biopsies' results.

\section{Discussion}

In this study, we established the positive patterns of staining, and subsequently determined the frequency of positive findings with the hope that more pathologists will utilize these two stains in their common practice to reliably diagnose invasion by colon carcinoma in colon polyps' biopsies. With the assist of IHC stains, cribriform architecture was identified in all cases of high grade lesions and invasive cancer and desmoplasia was identified in all cases of invasive cancer. In cases of colon polyp biopsies where $\mathrm{H} \& \mathrm{E}$ are hard to interpret, we recommend using CAM5.2 and caldesmon to help in making and/or confirming diagnosis.

Single cells in the lamina propria (Figure 1) were identified in $2 / 16(13 \%)$ of HGIN. In these two cases, single cells were not associated with desmoplasia, and the stroma surrounding these cells and nearby dysplastic glands were of lamina propria nature (Figure 1). Thus, we are hesitant in calling this invasion. And even if these single cells have been considered poorly differentiated cancer, in one study, follow-ups of 15 cases of poorly differentiated colon carcinoma with invasion restricted to lamina propria showed no evidence of residual tumor nor invasion [9], similar to the follow-ups of our two cases (see Result section above). Another possibility is that these single cells represent misplaced epithelium, although this term is generally reserved for noninvasive glands seen in submucosa.

Single cells were identified in $12 / 32$ (38\%) of IC. This is slightly lower than the reported rate of tumor budding frequencies reported in the past literatures $(41-75 \%[10,11])$. This could be due to the fact that 
Jia Y (2019) The roles of CAM5.2 and caldesmon IHC stains in challenge of diagnosing adenoma, intramucosal carcinoma, and invasive adenocarcinoma on colon biopsies
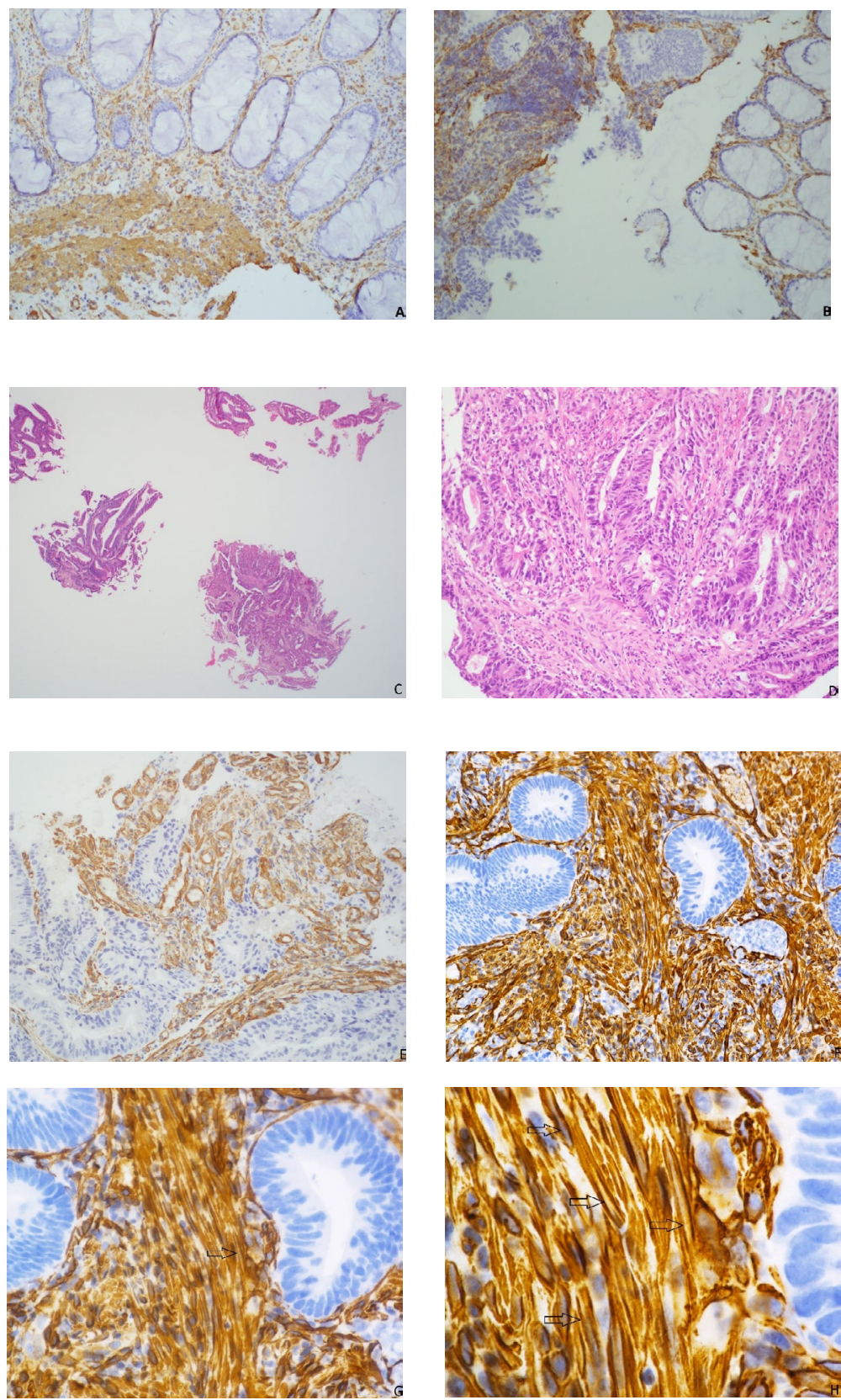

Figure 2. Utility of caldesmon. A. (20x) Caldesmon stained lamina propria (above) with faint brown thin lines surrounding small blood vessels while staining smooth muscle (below) as diffuse solid sheets. B. (20x) Caldesmon stained desmoplasia (left) strongly with clear space seen in cytoplasm while staining lamina propria (right) with thin lines C. (2x) H\&E low power gave an impression of a villotubular adenoma with a focus of high grade dysplasia (bottom right) D. (20x) H\&E high power of same focus showed the stroma to have mixed inflammatory cells and spindled cells, desmoplasia cannot be determined definitively E. (20x) Caldesmon stained the cytoplasm with clear spaces seen, confirming desmoplasia and diagnosis of invasive adenocarcinoma arising from villotubular adenoma F. (20x) Caldesmon stained the cytoplasm with the "tram-tracking appearance": The desmoplastic cells were closely packed and formed thickened bands parallel along the tumor cell borders. G. (40x) A clear straight line was seen running along the middle of the cytoplasm of a desmoplastic myofibroblast (arrow), consistent with desmoplasia. H. (100x) Clear straight lines were seen inside the cytoplasm of multiple desmoplastic cells (arrows)

we did not order CAM5.2 and caldesmon on clear-cut malignant cases. Tumor budding has been proven to be associated with worse prognosis in multiple papers. With this study, we can confirm that CAM5.2 is an excellent stain to evaluate for tumor budding.

Formation of desmoplasia, including epithelial and stromal interaction, is also frequently studied in colon cancer [12]. MMP-1, p53, E-cadherin, and collagen IV IHC stains have been used to confirm desmoplasia to distinguish adenomas with misplaced epithelium versus invasive adenocarcinoma [4]. Caldesmon, to the best of our knowledge, has not been evaluated in the purpose of confirming desmoplasia in colon cancer. We suspect caldesmon would also be useful in identifying misplaced epithelium as opposed to invasive adenocarcinoma in the submucosa.

The subtype of desmoplastic pattern at the tumor front can also influence prognosis of colon cancer [12]. In our experience, caldesmon works best at identifying desmoplasia, while AB/PAS may be a better stain to distinguish different types of desmoplasia, such as hyalinized collagen from myxoid stroma. 
Jia Y (2019) The roles of CAM5.2 and caldesmon IHC stains in challenge of diagnosing adenoma, intramucosal carcinoma, and invasive adenocarcinoma on colon biopsies

\section{Future studies}

We plan to study and utilize this combination of the two stains to differentiate reactive changes versus invasive malignancies in pancreatic tumors.

\section{References}

1. Fleming M, Ravula S, Tatishchev S (2012) Colorectal carcinoma: Pathologic aspects. $J$ Gastrointest Oncol 3: 153-173.

2. Conti J, Thomas G (2011) The role of tumour stroma in colorectal cancer invasion and metastasis. Cancers (Basel) 3: 2160-2168. [Crossref]

3. Wong HH, Chu P (2012) Immunohistochemical features of the gastrointestinal tract tumors. J Gastrointest Oncol 3: 262-284. [Crossref]

4. Yantiss R, Bosenberg M, Antonioli D (2002) Utility of MMP-1, p53, E-cadherin, and collagen IV immunohistochemical stains in the differential diagnosis of adenomas with misplaced epithelium versus adenomas with invasive adenocarcinoma. Am J Surg Pathol 26: 206-215.

5. Amezcua C, MacDonald H, Lum C (2006) Endometrial cancer patients have significant risk of harboring isolated tumor cells in histologically negative lymph nodes. Int J Gynecol Cancer 16: 1336-1341.
6. Perez-Montiel M, Plaza J, Dominguez-Malagon H (2006) Differential expression of smooth muscle myosin, smooth muscle actin, h-caldesmon, and calponin in the diagnosis of myofibroblastic and smooth muscle lesions of skin and soft tissue. Am J Dermatopathol 28: 105-111.

7. Nucci M, O'Connell J, Huettner P (2001) h-Caldesmon expression effectively distinguishes endometrial stromal tumors from uterine smooth muscle tumors. $\mathrm{Am} \mathrm{J}$ Surg Pathol 25: 455-463.

8. Roberts JA, Waters L, Ro JY, Zhai QJ (2014) Smoothelin and caldesmon are reliable markers for distinguishing muscularis propria from desmoplasia: a critical distinction for accurate staging colorectal adenocarcinoma. Int J Clin Exp Pathol 7: 792-796. [Crossref]

9. Lewin M, Fenton H, Burkart A (2007) Poorly differentiated colorectal carcinoma with invasion restricted to lamina propria (intramucosal carcinoma): a follow-up study of 15 cases. Am J Surg Pathol 31: 1882-1886.

10. Kazama S, Watanabe T, Ajioka Y (2006) Tumor budding at the deepest invasive margin correlates with lymph node metastasis in submucosal colorectal cancer detected by anticyokeratin antibody CAM5.2. Br J Cancer 94: 293-298.

11. Okuyama T, Oya M, Ishikawa $\mathrm{H}$ (2002) Budding as a risk factor for lymph node metastasis in pT1 or pT2 well-differentiated colorectal adenocarcinoma. Dis Colon Rectum 45: 628-634. [Crossref]

12. Ueno H, Kanemitsu Y, Sekine S (2007) Desmoplastic pattern at the tumor front defines poor-prognosis subtypes of colorectal cancer. Am J Surg Pathol 41: 1506-1512.

Copyright: (C2019 Jia Y. This is an open-access article distributed under the terms of the Creative Commons Attribution License, which permits unrestricted use, distribution, and reproduction in any medium, provided the original author and source are credited. 